\title{
Citação de artigos nacionais: a (des)valorização dos periódicos brasileiros
}

\section{Citing of national articles: the (de)valuation of brazilian journals}

\author{
Renan Kleber Costa Teixeira ${ }^{1}$; Tiago Santos Silveira ${ }^{2}$; Nara Macedo Botelho ${ }^{3}$; Andy Petroianu, TCBC-MG ${ }^{4}$
}

\author{
R E S U M O
}

\begin{abstract}
Objetivo: Avaliar se há preferência pela citação de periódicos estrangeiros em detrimento dos brasileiros, em três revistas brasileiras de cirurgia, em dois períodos distintos. Métodos: Foram avaliadas todas as referências dos artigos publicados nos anos de 2011 e 2007, pelas revistas Acta Cirúrgica Brasileira, Arquivos Brasileiros de Cirurgia Digestiva e Revista do Colégio Brasileiro de Cirurgiões, verificando a quantidade de artigos provenientes de revistas brasileiras e estrangeiras. Resultados: Foram analisadas 7343 referências dispostas em 348 artigos nas três revistas analisadas, desse total, 856 (11,65\%) eram de periódicos brasileiros. Não houve diferença entre as três revistas analisadas, nem entre os dois períodos analisados. Cento e onze (31,9\%) artigos não citaram artigo de periódico brasileiro e 36 (10,34\%), citaram mais os de brasileiros do que os de estrangeiros. Conclusão: há necessidade de o pesquisador brasileiro valorizar mais os periódicos brasileiros aumentando a citação do Brasil, sem uma xenofobia científica.
\end{abstract}

Descritores: Artigo de revista. Fator de impacto. Bibliografia como assunto.

\section{INTRODUÇÃO}

A pesquisa científica só expressa sua real contribuição à comunidade quando alcança seu objetivo final: a publicação ${ }^{1}$. Esta é de valor imensurável, visto que o registro do conhecimento foi o mais eficaz meio para transmitir informações. Culturas que não desenvolveram a escrita perderam-se no tempo, indicando que a transmissão do conhecimento não se perpetuou por longo período².

A publicação brasileira vem crescendo, nos últimos anos, de forma exponencial ${ }^{3}$. Na década de 1960, a média de publicações científicas publicadas em periódicos registrados no Information Sciences Institute (ISI), era de 52 artigos anuais. Na década de 1970 houve pouca mudança e a média cresceu para 64 artigos. Contudo, em 2001, os pesquisadores brasileiros publicaram cerca de 10.555 artigos em periódicos indexados, correspondendo a um crescimento de 165 vezes na publicação brasileira. No mesmo período, o crescimento mundial foi 2,18 vezes $^{4}$.

Esse crescimento é graças, em grande parte, às universidades publicas brasileiras, por meio dos programas de pós-graduação stricto sensu, que são os grandes centros formadores de pesquisa nacional ${ }^{5}$. Esses programas são avaliados pela Coordenação de Aperfeiçoamento de Pessoal de Nível Superior (CAPES). Um dos parâmetros utilizados para classificar a qualidade do programa de pósgraduação é a qualidade dos periódicos onde os artigos são publicados. Essa avaliação é realizada por meio do QUALIS do periódico ${ }^{6}$.

O QUALIS é uma medida indireta que avalia a qualidade do periódico, com base no fator de impacto, calculado de acordo com a quantidade de vezes que um artigo é citado por outros artigos ${ }^{7}$. Essa classificação utilizada pela CAPES é amplamente criticada, vista que não avalia a qualidade do artigo, mas o local onde este é publicado; além disso, essa avaliação desincentiva a publicação em periódicos nacionais, cujo valor de QUALIS é menor $8,9,10$.

Essa desvalorização dos periódicos brasileiros gera um viés muitas vezes despercebido por grande parte dos pesquisadores: a baixa citação de artigos publicados em revistas brasileiras. Na tentativa de publicar em revistas estrangeiras, utilizam-se artigos estrangeiros em detrimento dos publicados em periódicos brasileiros. Sendo que esses autores acabam por utilizar referencias internacionais até mesmo quando encaminham seus artigos para periódicos brasileiros, sob a justificativa da qualidade dos artigos estrangeiros ser superior ${ }^{11,12}$.

Contudo, na revisão de literatura realizada, não foi identificado artigo comprovando realmente essa maior citação de artigos internacionais em relação aos nacionais

Trabalho realizado na Universidade do Estado do Pará - Pará-BR.

1. Discente da Faculdade de Medicina - Universidade do Estado do Pará; 2. Professor mestre do Curso de Educação Física da Universidade do Estado do Pará; 3. Professora Associada do Departamento de Saúde Especializada da Universidade do Estado do Pará; 4. Professor Titular do Departamento de Cirurgia da Faculdade de Medicina da UFMG. 
nos periódicos brasileiros. Assim, o objetivo deste trabaIho foi avaliar se há uma preferência pela citação de periódicos de outras nações em detrimento dos brasileiros em três revistas brasileiras de cirurgia, em dois períodos distintos.

\section{MÉTODOS}

Este trabalho caracteriza-se como observacional e transversal. Foram analisadas as referências de três periódicos brasileiros de cirurgia (Acta Cirúrgica Brasileira, Arquivos Brasileiro de Cirurgia Digestiva e Revista do Colégio Brasileiro de Cirurgiões).

Nessas revistas foram analisados todos os artigos publicados nos anos de 2011 e 2007. Foram incluídos todos os artigos definidos como artigos originais. Os artigos classificados como: editoriais, revisão de literatura, relato de caso ou artigos sem referências, e aqueles em que mais de $75 \%$ das referências não eram artigos científicos foram excluídos da pesquisa. Os artigos dentro do critério de inclusão e exclusão foram analisados com base nas referências utilizadas pelos artigos brasileiros.

O protocolo de pesquisa avaliou a quantidade total de referências utilizadas, sendo desconsideradas da contagem referências de livros, páginas de internet ou citação de citação (apud). Verificaram-se quantas referências eram provenientes de periódicos brasileiros e estrangeiros e a relação entre artigos de periódicos brasileiros com os artigos de periódicos estrangeiros.

Foi utilizado o teste ANOVA para avaliar se houve mudança entre os dois períodos em cada revista e se havia diferença entre as revistas. Foi adotado $p<0,05$ para que os dados fossem considerados diferentes.

\section{RESULTADOS}

Foram analisadas 7343 referências dispostas em 348 artigos nas três revistas analisadas, tendo uma média de $21,1 \pm 8,55$ citações por artigo. Destas $856(11,65 \%)$ eram referentes a periódicos brasileiros, correspondendo a uma média de 2,46 $\pm 3,16$ citações por artigo.

Em relação aos dois anos avaliados, em 2011, foram citadas 4094 referências, em 189 artigos, tendo uma média de 21,66 \pm 8,18 citação por artigo, dessas 504 $(12,31 \%)$ eram referentes a periódicos brasileiros, perfazendo uma média de 2,66 $\pm 3,28$ citações por artigo. No ano de 2007, os 159 artigos analisados apresentaram 3249 referências, tendo uma média de 20,43 \pm 8,96 referências por artigo, deste total, 352 citações eram de periódicos brasileiros, correspondendo a 2,21 $\pm 3,01$ citações por artigo. Não houve diferença entre os dois períodos analisados $(p=0,1492)$.

Nos Arquivos Brasileiros de Cirurgia Digestiva foram estudados 86 artigos que apresentaram 1795 refe- rências, onde 238 (13,26\%) eram referentes a periódicos brasileiros. Quando verificado por ano, em 2011, houve 1081 referências, das quais 151 (13,96\%) eram provenientes de periódicos brasileiros; em 2007, das 714 referências utilizadas, 87 (12,46\%) eram citações de artigos brasileiros.

Na Revista do Colégio Brasileiro de Cirurgiões, 2301 referências foram identificadas em 105 artigos, 277 $(12,04 \%)$ oriundas de periódicos brasileiros. Em 2011, essa revista publicou 54 artigos que apresentaram 154 referências a artigos brasileiros de um total de 1205 . Em relação ao ano de 2007, 1096 referências foram utilizadas em 51 artigos que citaram $123(11,22 \%)$ artigos de periódicos brasileiros.

Na Acta Cirúrgica Brasileira, 157 artigos apresentaram 3247 referências, das quais 341 (10,5\%) foram de citações a artigos de periódicos brasileiros. Em 2011, os 87 artigos analisados apresentaram 1808 referências, dentre as quais 199 (11\%) eram referências de artigos brasileiros. Em 2007, das 1439 referências, presentes em 70 artigos, 142 (9,86\%) foram de artigos brasileiros.

Não houve diferença entre a quantidade de citações de artigos publicados nestas revistas entre os dois períodos analisados, nem em relação à quantidade das revistas.

Quando analisada a relação entre a citação de periódicos brasileiros por periódicos de outros países, percebe-se que 111 (31,9\%) dos periódicos estudados não citaram artigo publicados nas revistas brasileiras, sendo este valor, em 2011, de 31,22\% e, em 2007, de 32,7\%, sem diferença significativa. Pode-se perceber que apenas 13 $(3,74 \%)$ artigos utilizaram mais artigos publicados no Brasil do que os publicados em outros países, sendo essa proporção, em 2011, de 4,75\% e, em 2007, de 2,52\%, sem diferença significativa.

A maioria dos artigos analisados $(45,11 \%)$ apresentou uma relação entre artigos nacionais por internacionais entre $0,01 \%$ e $24,99 \%$ (Tabela 1 ).

\section{DISCUSSÃO}

O modelo de avaliação de um artigo é baseado no fator de impacto do periódico onde este foi publica$\mathrm{do}^{9,12}$. O fator de impacto é calculado com base na relação entre total de citação que o periódico recebeu em dois anos pela quantidade de artigos publicados nesse período, sendo, portanto, uma medida que não avalia diretamente os artigos publicados, mas o conjunto dos artigos publicados em determinado período ${ }^{13}$.

Mesmo havendo várias críticas a esse modelo, ele tornou-se "consagrado" devido à adoção pela CAPES para avaliar a qualidade das publicações das pós-graduações stricto sensu $u^{10,11}$.

Nesta pesquisa constatou-se que, nas revistas de cirurgia, aproximadamente, para cada artigo publicado em 
Tabela 1 - Relação entre artigos nacionais por internacionais por revista e ano analisado.

\begin{tabular}{|c|c|c|c|c|c|c|c|c|c|c|c|c|}
\hline \multirow[t]{3}{*}{ Percentil } & \multicolumn{6}{|c|}{2011} & \multicolumn{6}{|c|}{2007} \\
\hline & \multicolumn{2}{|c|}{$A B C D$} & \multicolumn{2}{|c|}{$\mathrm{RCBC}$} & \multicolumn{2}{|c|}{$A C B$} & \multicolumn{2}{|c|}{$A B C D$} & \multicolumn{2}{|c|}{$\mathrm{RCBC}$} & \multicolumn{2}{|c|}{$A C B$} \\
\hline & $\mathrm{N}$ & $\%$ & $\mathrm{~N}$ & $\%$ & $\mathrm{~N}$ & $\%$ & $\mathrm{~N}$ & $\%$ & $\mathrm{~N}$ & $\%$ & $\mathrm{~N}$ & $\%$ \\
\hline $0 \%$ & 11 & 22,92 & 15 & 27,78 & 33 & 37,93 & 13 & 34,21 & 15 & 29,41 & 24 & 34,29 \\
\hline $0,01 \%-24,99 \%$ & 21 & 43,75 & 28 & 51,85 & 33 & 37,93 & 17 & 44,74 & 24 & 47,06 & 34 & 48,57 \\
\hline $25 \%-49,99 \%$ & 9 & 18,75 & 3 & 5,56 & 12 & 13,79 & 3 & 7,89 & 8 & 15,68 & 9 & 12,86 \\
\hline $50 \%-74,99 \%$ & 2 & 4,17 & 3 & 5,56 & 5 & 5,75 & 3 & 7,89 & 1 & 1,96 & 2 & 2,86 \\
\hline $75 \%-99,99 \%$ & 2 & 4,17 & 1 & 1,85 & 1 & 1,15 & 0 & 0 & 1 & 1,96 & 0 & 0 \\
\hline $100 \%$ & 1 & 2,08 & 0 & 0 & 0 & 0 & 1 & 2,63 & 0 & 0 & 0 & 0 \\
\hline$>100 \%$ & 2 & 4,17 & 4 & 7,41 & 3 & 3,45 & 1 & 2,63 & 2 & 3,92 & 1 & 1,43 \\
\hline Total & 48 & 100 & 54 & 100 & 87 & 100 & 38 & 100 & 51 & 100 & 70 & 100 \\
\hline
\end{tabular}

Fonte: Protocolo de pesquisa

$A B C D$ - Arquivos Brasileiros de Cirurgia Digestiva; $R C B C$ - Revista do Colégio Brasileiro de Cirurgião; ABC - Acta Cirúrgica Brasileira. $p=0,8549$ (ANOVA)

periódico nacional cita-se nove internacionais. Essa prática não pode ser considerada como errada, contudo é um desprestígio para os periódicos nacionais. Se os próprios pesquisadores brasileiros não citam os periódicos nacionais, o que esperar dos pesquisadores de outros países?

O fato de não ter ocorrido mudança nas características de citação nos dois períodos analisados mostra a perpetuação do ciclo de desprestígio nacional. Os próprios orientadores de programas de pós-graduação ensinam a valorização dos artigos internacionais em detrimento dos nacionais.

Entre os dois períodos estudados houve um crescimento no fator de impacto dos periódicos brasileiros, devido às políticas editoriais bem conduzidas para a indexação em bases bem conceituadas. Esse crescimento deve-se à elevada qualidade dos artigos publicados nesses periódicos, mostrando que a publicação brasileira possui elevado grau de qualidade.

Um exemplo que pode comprovar esse fato é o estudo de Marques, que, ao estudar a produção brasileiros entre 1994 e 2003 encontrou 248 artigos com mais de cem citações cada, comprovando que não é a qualidade dos artigos o fator limitante ao crescimento dos periódicos brasileiros $^{14}$

Contudo, se os pesquisadores brasileiros preferirem citar os artigos de periódicos estrangeiros em vez dos brasileiros, a disparidade existente atualmente irá crescer $^{15}$, devido à exteriorização dos bons artigos brasilei$\operatorname{ros}^{12,16}$, são poucos os pesquisadores estrangeiros que citam revistas brasileiras.

A qualidade da pesquisa brasileira tem crescido no cenário internacional, porém o mesmo não ocorre com relação à editoração nacional. Contudo, não é proposto neste estudo uma xenofobia científica, mas uma valorização dos periódicos nacionais ${ }^{16}$. É de senso comum que valiosos dados científicos são publicados fora do país e que estes devem ser citados, porém, é inaceitável que 31,9\% dos artigos dos periódicos analisados não citem, pelo menos, um artigo publicado no país.

É necessária uma conscientização dos pesquisadores brasileiros para que publiquem seus principais estudos nas revistas brasileiras, para que elas melhorem seu fator de impacto e forcem os pesquisadores estrangeiros a lerem os periódicos brasileiros se desejarem conhecer a ciência de nosso país. Na China, os pesquisadores publicam em diversos periódicos estrangeiros, porém seus principais resultados são publicados nos periódicos chineses, forçando os demais pesquisadores a lerem e citarem os periódicos desse país ${ }^{12}$.

Uma prova de que é possível utilizar mais os nossos periódicos é que 15 artigos (4,31\%) analisados utilizaram mais de $50 \%$ de artigos nacionais e 36 (10,34\%) artigos utilizaram mais artigos nacionais do que estrangeiros. Em apenas um artigo todas as referencias eram provenientes de periódicos brasileiros, mostrado que, se os pesquisadores desejarem, é possível utilizar periódicos nacionais sem prejudicar a qualidade do artigo publicado.

Outro mecanismo que pode ser utilizado para ampliar a citação de periódicos brasileiros é por meio dos revisores peer review, que podem sugerir artigos nacionais para os autores, reforçando a própria revista.

É necessária uma conscientização dos pesquisadores para publicarem seus bons trabalhos em revistas brasileiras, valorizando-as, em uma política de incentivo à citação dos periódicos brasileiros. 


\section{A}

Objective: To evaluate whether there is a preference for foreign periodicals over Brazilian one. Methods: We evaluated all references of articles published in the years 2011 and 2007 in the journals Acta Cirúrgica Brasileira, Arquivos Brasileiros de Cirurgia Digestiva and the Journal of the Brazilian College of Surgeons, verifying the quantity of papers from Brazilian and foreign publications. Results: We analyzed a total of 7,343 references arranged in 348 articles in those three journals; of these, 856 (11.65\%) were from Brazilian journals. There was no difference between the three magazines, nor between the two periods. One hundred and eleven (31.9\%) articles did not cite any Brazilian journal article and 36 (10.34\%) cited Brazilian articles more than foreign ones. Conclusion: There is need for the Brazilian researcher to appreciate more the Brazilian journals, increasing citation from Brazil, without a scientific xenophobia.

Key words: Journal article. Impact factor. Bibliography as topic.

\section{REFERÊNCIAS}

1. Rosas $P$, Guimarães $C A$, Júdice LF, Ferreira CAC, Válio EBM Descritores em ciências da saúde nas teses e dissertações de mestrado, na área de doenças respiratórias. Acta cir bras. 1999;14(1):43-6.

2. Timi JRR. A importância do uso dos descritores nas publicações médicas. J vasc bras. 2005;4(2):114-5.

3. Brasil. Ministério da Ciência e Tecnologia. Participação percentual do número de artigos brasileiros publicados em periódicos científicos indexados pela Thomson/ISI e Scopus em relação ao mundo, 1996-2011. 2012. Acessado em: 19 de junho de 2012. Disponível em: http://www.mct.gov.br/index.php/content/view/5711.html.

4. Guimarães JA. A pesquisa médica e biomédica no Brasil: comparações com o desempenho científico brasileiro e mundial. Ciênc saúde coletiva. 2004:9(2):303-27.

5. Demo P. Qualidade e pesquisa na universidade. RBDEPA. 2009;1(1):52-64.

6. Silva AL. Capes e revista de impacto. Rev Col Bras Cir. 2011;38(6):371.

7. Lucena AF, Tibúrcio RV. QUALIS periódicos: visão do acadêmico na graduação médica. Rev Assoc Med Bras. 2009;55(3):247-8.

8. Rocha-e-Silva M. Qualis 2011-2013: os três erres. Clinics. 2010;65(10):935-6.

9. Rocha-e-Silva M. O Novo Qualis, que não tem nada a ver com a ciência do Brasil: carta aberta ao presidente da CAPES. Clinics. 2009;64(8):721-4
10. Editores Científicos de Revistas Médicas Brasileiras. Classificação dos periódicos no Sistema QUALIS da CAPES - A mudança dos critérios é urgente! Arq Bras Endocrinol Metab. 2010;54(1):3-5.

11. Goffi FS. Um pouco de nacionalismo nas publicações científicas brasileiras. Rev Col Bras Cir. 2007;34(4):212.

12. Petroianu A. Perversidade contra a publicação médica no Brasil. Rev Col Bras Cir. 2011; 38(5):290-1

13. Thomson Reuters. ISI Web of Knowledge Web site. 2011; Acessado em: 10 de junho de 2012. Disponivel em: http://wokinfo.com/.

14. Marques F. Em que somos bons? Pesquisa FAPESP. 2007;132:23-5.

15. Goldenberg S. Em que somos bons? Acta cir bras 2007;22(3):160-1.

16. Silva MR. A publicação de ciência em países em desenvolvimento. Os novos atores. Clinics. 2006;61(5);375-80.

Recebido em 01/07/2012

Aceito para publicação em 31/07/2012

Conflito de interesse: nenhum

Fonte de financiamento: nenhum

\section{Como citar este artigo:}

Teixeira RKC, Silveira TS, Botelho NM, Petroianu A. Citação de artigos nacionais: a (des)valorização dos periódicos brasileiros. Rev Col Bras Cir. [periódico na Internet] 2012; 39(5). Disponível em URL: http:// www.scielo.br/rcbc

Endereço para correspondência:

Renan Kleber Costa Teixeira

E-mail: renankleberc@hotmail.com 